\title{
Genetic and pharmacological analysis of prostanoid receptor function
}

\author{
Shuh Narumiya ${ }^{1}$ and Garret A. FitzGerald ${ }^{2}$ \\ ${ }^{1}$ Department of Pharmacology, Kyoto University Faculty of Medicine, Kyoto, Japan \\ ${ }^{2}$ Center for Experimental Therapeutics, University of Pennsylvania, Philadelphia, Pennsylvania, USA \\ Address correspondence to: Shuh Narumiya, Kyoto University, Faculty of Medicine, Department of Pharmacology, Yoshida, \\ Sakyo-ku, Kyoto 606, Japan. Phone: 81-75-753-4392; Fax: 81-75-753-4693; E-mail: snaru@mfour.med.kyoto-u.ac.jp. \\ J. Clin. Invest. 108:25-30 (2001). DOI:10.1172/JCI200113455.
}

When tissues are exposed to diverse physiological and pathological stimuli, arachidonic acid is liberated from membrane phospholipids and is converted to prostanoids, including the prostaglandins (PGs) and the thromboxanes (Tx's), by the action of cyclooxygenase (COX). The COX reaction results in the formation of an unstable endoperoxide intermediate, $\mathrm{PGH}_{2}$, which, in turn, is metabolized to $\mathrm{PGD}_{2}, \mathrm{PGE}_{2}, \mathrm{PGF}_{2 \alpha}$, $\mathrm{PGI}_{2}$, and $\mathrm{TxA}_{2}$ by cell-specific isomerases and synthases. Prostanoids thus formed are immediately released outside of the cell, with little if any of the product remaining in the cell. Because they are either chemically or metabolically unstable, it is believed that prostanoids work only locally, near their site of production. $\mathrm{PGI}_{2}$ and $\mathrm{TxA}_{2}$ spontaneously degrade into inactive compounds under physiological conditions, and other PGs are enzymatically inactivated during a single passage through the lung. In addition, $\mathrm{PGD}_{2}$ and $\mathrm{PGE}_{2}$ are slowly dehydrated in biological fluids containing serum albumin to yield the cyclopentenone PGs, $\mathrm{PGA}_{2}$ and $\mathrm{PGJ}_{2}$, which contain an $\alpha, \beta$-unsaturated ketone.

Although prostanoids are generally regarded as hydrophobic compounds, they do not permeate the cell membrane freely. One PG transporter (PGT), a member of the organic anion transporter polypeptide fami$l y$, has been identified and is found in a limited range of cells, where it is subject to regulation by humoral and physical stimuli. Indeed, vascular expression of the PGT appears to account for clearance of most classes of PGs when these compounds are infused into an animal and enter its pulmonary circulation. Indeed, $\mathrm{PGI}_{2}$, the only PG that is not a substrate for PGT, is also unique in that it is not subject to clearance during traversal of the pulmonary circulation. However, there is strong evidence that other transport systems mediate the uptake of at least some prostanoids, since despite the restricted expression of PGT, cyclopentenone PGs are efficiently transported into most cells. For more on transport and metabolism of prostanoids, see Fitzpatrick and Soberman (this Perspective series, ref. 1).

Prostanoids, with the possible exception of the cyclopentenone PGs, are believed to exert their actions via membrane receptors on the surface of target cells. Indeed, a family of membrane receptors mediating their actions has been characterized and cloned, and knockout mice deficient in each of their genes have been developed. In addition, highly selective agonists and antagonists for the cloned receptors have been developed. Nuclear actions of prostanoids have also been reported and putative nuclear prostanoid receptors have been proposed, particularly for cyclopentenone PGs. In this article, we describe the current status of the studies on membrane prostanoid receptor biology and discuss the clinical potential of receptor-selective drugs. We then critically examine the current evidence for activation of nuclear receptors by prostanoids.

\section{Membrane prostanoid receptors:}

\section{studies in knockout mice}

Eight types and subtypes of membrane prostanoid receptors are conserved in mammals from mouse to human (2): the PGD receptor (DP), four subtypes of the PGE receptor (EP1, EP2, EP3, and EP4), the PGF receptor (FP), the PGI receptor (IP), and the TxA receptor (TP). All are G protein-coupled rhodopsin-type receptors with seven transmembrane domains, and each is encoded by different genes. In addition, there are several splice variants of the EP3, FP, and TP receptors, which differ only in their C-terminal tails. Among the eight types and subtypes, the IP, DP, EP2, and EP4 receptors mediate a cAMP rise and have been termed "relaxant" receptors, whereas the TP, FP, and EP1 receptors induce calcium mobilization and constitute a "contractile" receptor group. The remaining receptor, EP3, induces a decline in cAMP levels and has been termed the "inhibitory" receptor. However, the effects of prostanoids on these $\mathrm{G}$ protein-coupled signaling pathways may change as a function of ligand concentration or structure. Thus, while $\mathrm{PGI}_{2}$ analogs ligate the IP and activate adenylate cyclase via $\mathrm{G}_{\mathrm{s}}$ in a dosedependent manner, higher concentrations of the ligand couple the IP to phospholipase $\mathrm{C}$ activation and calcium mobilization (3), probably via $\mathrm{G}_{\mathrm{q}}$. Similarly, activation of the $\mathrm{FP}$ in cardiomyocytes by either $\mathrm{PGF}_{2 \alpha}$, its cognate ligand, or $8,12-i s o-\mathrm{PPF}_{2 \alpha}-\mathrm{III}$, a $\mathrm{PGF}_{2 \alpha}$ isomeric 
Table 1

Major phenotypes of mice deficient in prostanoid receptors

\begin{tabular}{|c|c|c|}
\hline Disrupted gene & Phenotypes & Reference \\
\hline$D P$ & Decreased allergic responses in ovalbumin-induced bronchial asthma & 8 \\
\hline EP1 & Decreased aberrant foci formation to azoxymethane & 17 \\
\hline \multirow[t]{5}{*}{$E P 2$} & Impaired ovulation and fertilization & $25,26,40$ \\
\hline & Salt-sensitive hypertension & 25,26 \\
\hline & Vasopressor or impaired vasodepressor response to intravenous $\mathrm{PGE}_{2}$ & 41,42 \\
\hline & Loss of bronchodilation $\mathrm{PGE}_{2}$ & 43 \\
\hline & Impaired osteoclastogenesis in vitro & 14 \\
\hline \multirow[t]{4}{*}{ EP3 } & Impaired febrile response to pyrogens & 7 \\
\hline & Impaired duodenal bicarbonate secretion and mucosal integrity & 44 \\
\hline & Enhanced vasodepressor response to intravenous infusion of $\mathrm{PGE}_{2}$ & 41 \\
\hline & Disappearance of indomethacin-sensitive urine diluting function & 45 \\
\hline \multirow[t]{3}{*}{ EP4 } & Patent ductus arteriosus & 46,47 \\
\hline & Impaired vasodepressor response to intravenous infusion of $\mathrm{PGE}_{2}$ & 41 \\
\hline & Decreased inflammation bone resorption & 12,13 \\
\hline$F P$ & Loss of parturition & 48 \\
\hline \multirow[t]{3}{*}{ IP } & Thrombotic tendency & 5 \\
\hline & Decreased inflammatory swelling & 5,49 \\
\hline & Decreased acetic acid writhing & 5 \\
\hline$T P$ & Bleeding tendency and resistance to thromboembolism & 20 \\
\hline
\end{tabular}

product of lipid peroxidation, results in activation of distinct, as well as overlapping, signaling pathways (4).

The existence of four subtypes of the receptor for $\mathrm{PGE}_{2}$ is remarkable, given that the other prostanoids each have only a single receptor. The homology of amino acid sequences between different types of the receptors within each functional group is much higher than that found among the four PGE receptor subtypes. The phylogenetic tree derived from receptor homologies indicates that prostanoid receptors originated from the primitive PGE receptor, from which the subtypes of the PGE receptor then evolved. Other PGs and Tx receptors subsequently evolved from functionally related PGE receptor subtypes by gene duplication. This evolutionary history suggests that the different paralogues play distinct physiological roles.

The roles of PGs in various physiological and pathophysiological processes have thus far been suggested by comparing the effects of aspirin-like drugs with those of each prostanoid added exogenously. However, such studies do not clearly indicate which type of prostanoid and which class of prostanoid receptor is involved in a given process, nor how critical the actions of prostanoids might be. To address these questions, we and others have generated and analyzed mice deficient in each prostanoid receptor. The reported findings of these knockout mice are summarized in Table 1. Here we discuss the significance of some of these findings. Inflammation and pain. Vasodilation and pain generation are two classic features of acute inflammation to which prostanoids appear to contribute. Aspirin-like drugs suppress these responses, and $\mathrm{PGE}_{2}$ and $\mathrm{PGI}_{2}$ can mimic these actions. Murata et al. (5) employed carrageenan-induced paw swelling and acetic acid-induced writhing as models for acute inflammation and pain, respectively, and showed that both responses are completely absent in IP-deficient mice. This study clearly demonstrated that $\mathrm{PGI}_{2}$, acting on the IP, works as a physiological mediator of these responses. However, it remains to be seen whether $\mathrm{PGI}_{2}$ and the IP play important roles in other types of inflammation and pain. The profiles of prostanoids generated in an inflammatory site change during the course of inflammation and are also dependent on the stimulus and site of inflammation (6). In pain, PGs are involved not only in hyperalgesia, an increased sensitivity to a painful stimulus, but also in allodynia, a pain response to a usually nonpainful stimulus. The latter condition is frequently seen in neuropathic pain and is thought to occur in the spinal cord, while the former is caused by sensitizing the free end of pain neurons in peripheral inflammation. It is interesting that the primary sensory neurons in the dorsal root ganglion express several types of prostanoid receptor mRNAs, including IP, EP1, EP3, and EP4 (2). The contribution of receptors other than IP to pain generation has not been determined.

Fever and other manifestations of systemic illness. Systemic illness is associated with fever, adrenocorticotropic hormone release, loss of appetite and libido, decreased locomotion, and the induction of slow-wave sleep. Because of the antipyretic action of aspirin-like drugs, it has long been speculated that prostanoids contribute to fever generation, but the identity of the prostanoids involved has remained elusive. Ushikubi et al. (7) clarified this issue by showing that EP3-deficient mice fail to respond to either exogenous or endogenous pyrogens. A similar approach might be adopted to elucidate the mechanism of other features of systemic illness. For instance, given the role of $\mathrm{PGD}_{2}$ in sleep cycles, one might determine the contribution of $\mathrm{PGD}_{2}$ to the drowsiness associated with systemic illness using DP knockout mice (2).

Allergy and immunity. The roles of prostanoids in allergy and immunity have been less well defined than those in acute inflammation, in part because the effects of aspirin and related drugs are far less marked. Nevertheless, allergic responses are associated with an increase in prostanoid formation. For example, $\mathrm{PGD}_{2}$ is a major 
prostanoid generated by mast cells upon allergen challenge and is produced abundantly in allergic diseases such as asthma, allergic dermatitis, and conjunctivitis. Little attention has been paid to $\mathrm{PGD}_{2}$ 's roles in allergy, but recently, Matsuoka et al. (8) examined this issue by subjecting DP-deficient mice to ovalbumin-induced allergic asthma. They found a marked reduction in the airway inflammation, obstruction, and hypersensitivity in DP-deficient animals, suggesting that $\mathrm{PGD}_{2}$, acting via the $\mathrm{DP}$, works as a mediator of allergy.

This finding raises anew the question of why aspirin is not beneficial in allergy and can even precipitate asthmatic attacks in certain individuals. We suspect that other prostanoids normally antagonize the action of $\mathrm{PGD}_{2}$, so that nonsteroidal anti-inflammatory drug (NSAID) treatment will have complex effects on the disease pathway. There may be multiple actions (cooperative and antagonistic) of prostanoid receptor ligands on immune cells. In vitro, $\mathrm{PGE}_{2}$ shows strong immunosuppressive activity on T cells and acts with LPS or IL-4 on B cells to facilitate IgE class switching. A variety of prostanoid receptors, including EP subtypes, the IP, and the TP, are expressed in T cells of mice, and prostanoids have been implicated in T cell development (9). Indeed, the membrane receptor CRTH2, which is more closely related to the $\mathrm{N}$-formyl peptide receptor superfamily than to the other prostanoid receptors, is preferentially expressed in Th2 cells and has been shown to mediate chemotactic responses to PGD (10). The potential importance of prostanoids in the regulation of the immune response is reviewed by Koller and colleagues elsewhere in this Perspective series (11).

Bone resorption and formation. $\mathrm{PGE}_{2}$ is known to have bone-resorptive activity and to mediate bone resorption induced by cytokines and LPS. There are three reports using the knockout mice to address this issue. Sakuma et al. (12) and Miyaura et al. (13) reported impaired osteoclast formation in cells cultured from EP4-deficient mice. Interestingly, not only $\mathrm{PGE}_{2}$-dependent but also IL-1-dependent osteoclastogenesis are affected by the EP4 mutation. On the other hand, Li et al. (14) reported that the osteoclastogenic response to $\mathrm{PGE}_{2}$, parathyroid hormone, and 1,25-dihydoxyvitamin $\mathrm{D}$ in vitro is reduced significantly in culture of cells from EP2-deficient mice. This apparent discrepancy is likely to reflect redundant roles of the two relaxant PGE receptor subtypes. Both Japanese groups found a small but significant $\mathrm{PGE}_{2}$-dependent response in EP4-deficient mice $(12,13)$, and $\mathrm{Li}$ et al. (14) reported a further decrease in osteoclastogenesis when an EP4-selective antagonist was added to culture of EP2-deficient cells. Redundant roles for prostanoid receptors of the relaxant class are also seen in macrophages, where EP2, EP4, and IP regulate production of cytokines in a similar fashion (15). In addition to inducing bone resorption, exogenous $\mathrm{PGE}_{2}$ can also induce bone formation, although the receptor mediating this latter response has not been identified. It also remains to be seen in what physiological contexts the metabolic and/or anabolic $\mathrm{PGE}_{2}$ actions on bone are important.

Cancer and angiogenesis. There is now enough evidence accumulating to suggest the involvement of both COX isoforms in colon carcinogenesis, as outlined by Smith and Langenbach in this series (16). However, whether and how prostanoids are involved in this process is less certain. Knockout mice deficient in each prostanoid receptor provide a nice opportunity to test this issue. Indeed, Watanabe et al. (17) treated several lines of the knockout mice with a colon carcinogen, azoxymethane, and found significant suppression of aberrant crypt foci in EP1-deficient mice. Although they could reproduce their findings by administration of a selective EP1 antagonist to wild-type mice, the suppression was limited in both cases, suggesting the possible involvement of other receptors and/or other mechanisms. Moreover, aberrant crypt formation represents an initial step in carcinogenesis, and many events precede the development of colon cancer. It is very likely that prostanoids are also involved in other steps and mechanisms, as nicely described by Prescott in a recent issue of the JCI (18). Candidates for other PG-involved steps have been suggested by in vitro analysis and include the prevention of apoptosis of transformed cells and the stimulation of tumor-associated angiogenesis.

Cardiovascular effects of eicosanoids. The cardioprotective effects of low doses of aspirin are attributed to its ability to inhibit cumulatively COX-1 in platelets, as outlined in the contribution from Patrono and colleagues in this series (19). The principle vasoactive product of COX-1 in platelets is $\mathrm{TxA}_{2}$. There are two TP splice variants, but only the TP $\alpha$ isoform is expressed in mice. Interestingly, mice deficient in the TP $\alpha$ exhibit a mild bleeding tendency and resistance to platelet aggregation by TP agonists (20), consistent with the effects of TP antagonists administered to humans and with the observation that only the TP $\alpha$ isoform is translated in human platelets (21). Both isoforms are detectably expressed in endothelial and vascular smooth muscle cells (22), and directed overexpression of the TP $\beta$ isoform in the vasculature results in placental ischemia during pregnancy and a phenotype reminiscent of intrauterine growth retardation. Tx biosynthesis increases during pregnancy, a state of hemostatic activation, and suppression of $\mathrm{Tx}$ formation in the mice rescues the phenotype (23). In addition to its vasoconstrictor properties, $\mathrm{TxA}_{2}$ is a potent mitogen of vascular smooth muscle cells. Thus, TP antagonism or TP deletion decreases the vascular proliferative response to catheter-induced injury in the mouse, and TP antagonism retards atherogenesis (24).

Several eicosanoids, including $\mathrm{PGE}_{2}$ and $\mathrm{PGI}_{2}$, may contribute to regulation of systemic blood pressure. 
Table 2

Inhibition constants ( $\mathrm{nM}$ ) of cloned prostanoid receptors for conventional PG analogs and newly developed compounds

\begin{tabular}{|c|c|c|c|c|c|c|c|c|}
\hline Compounds & DP & EP1 & EP2 & EP3 & EP4 & FP & IP & TP \\
\hline \multicolumn{9}{|l|}{ Conventional PG analogs } \\
\hline Iloprost & $>10000$ & 21 & 1600 & 22 & 2300 & $>10000$ & 10 & $>10000$ \\
\hline Carbacyclin & $>10000$ & $>10000$ & 1600 & 31 & 2300 & 1200 & 110 & $>10000$ \\
\hline 17-Phenyl-PGE 2 & $>10000$ & 14 & $>10000$ & 4 & 1000 & 60 & $>10000$ & $>10000$ \\
\hline \multicolumn{9}{|c|}{ Newly developed compounds } \\
\hline \multicolumn{9}{|l|}{ EP1 antagonists } \\
\hline ONO-8713 & $>10000$ & 0.3 & 3000 & 1000 & $>10000$ & 1400 & 10000 & 10000 \\
\hline \multicolumn{9}{|l|}{ EP agonists } \\
\hline ONO-AE1-259 & $>10000$ & $>10000$ & 3 & $>10000$ & $>10000$ & $>10000$ & $>10000$ & $>10000$ \\
\hline ONO-AE-248 & $>10000$ & $>10000$ & 3700 & 8 & 4200 & $>10000$ & $>10000$ & $>10000$ \\
\hline ONO-AE-329 & $>10000$ & $>10000$ & 2100 & 1200 & 10 & $>10000$ & $>10000$ & $>10000$ \\
\hline \multicolumn{9}{|l|}{ DP agonist } \\
\hline L- 644,698 & 0.9 & $>25400$ & 267 & 3730 & 9280 & $>25400$ & $>25400$ & $>25400$ \\
\hline
\end{tabular}

However, differences in the expression of a hypertensive phenotype in mice lacking the EP2 receptor (25, 26) have highlighted the importance of genetic background and gender in conditioning this response. Further insights into the role of prostanoids in hemodynamics, reproduction, and renal function afforded by knockout mice are summarized in Table 1.

\section{Clinical potential of}

prostanoid receptor-selective drugs

Cloning of members of the prostanoid receptor family has naturally facilitated reassessment of previously developed receptor-active compounds, as well as the development of new drugs. Using a panel of Chinese hamster ovary cells stably expressing each cloned receptor, Kiriyama et al. (27) examined the binding properties of a series of PGs and PG analogs used in pharmacological analysis. This analysis revealed that most of these compounds exhibit no absolute selectivity. For example, as shown in Table 2, two $\mathrm{PGI}_{2}$ analogs, iloprost and carbacyclin, indeed bind to IP with good affinities, but they also bind to EP3 with similar or better affinities, and iloprost binds EP1 as well. 17-phenyl-PGE ${ }_{2}$, which is used as an EP1 agonist in many analyses, binds to EP3 with a better affinity than that to EP1 and to FP with a reasonable affinity. The considerable cross-reactivity exhibited by these conventional PG analogs is probably due to the fact that they have not been selected for by specific binding assays, but were screened for by bioassay on native tissues and cells that express more than one type of prostanoid receptor. Indeed, Okada et al. (28) recently compared the responses to PG analogs on gastric fundus and ileum from mice deficient in individual PG receptors. This study showed that contraction of these tissues in response to $\mathrm{PGE}_{2}$ is mediated by three receptors: EP1, EP3, and FP. Recently, a new generation of compounds has been reported, which have been assessed on a complete set of cloned receptors
(Table 2). As shown, they are highly selective for each receptor. Indeed, these compounds exert pharmacological actions consistent with the phenotypes of mice deficient in the corresponding receptor. These drug studies confirm the involvement of EP1 in precancerous lesions evoked by chemical carcinogens (17), the role of EP4 in bone resorption $(12,13)$, the regulation of alkali secretion by EP 3 , and the suppression of TNF- $\alpha$ production by EP2 and EP4 (15).

What, then, is the clinical potential of these selective agonists and antagonists, specifically when compared with selective inhibitors of COX-2 or with traditional NSAIDs, such as aspirin? Aspirin inhibits both COX isozymes but preferentially targets COX-1 when used in low doses $(16,29)$. Low-dose aspirin is effective in the prevention of myocardial infarction and stroke but can still result in gastric toxicity (19). Indeed, when lowdose aspirin is given to individuals who have not previously suffered an event, the number of strokes and myocardial infarctions prevented is almost precisely balanced by the number of major gastrointestinal events caused by the drug. COX-2 inhibitors, such as rofecoxib and celecoxib, theoretically bypass the consequences of inhibiting COX-1-dependent prostanoids relevant to homeostatic functions, such as hemostasis and gastric cytoprotection. Indeed, at doses that exhibit similar efficacy in the treatment of arthritis, rofecox$\mathrm{ib}$, the most selective compound currently approved for clinical use, results in considerably less gastrotoxicity than the isoform-nonspecific inhibitor naproxen.

However, although COX-2 is typically induced by inflammatory stimuli, its products may also play a protective effect in some settings. Thus, COX-2 appears to be the predominant source of $\mathrm{PGI}_{2}$ formation by the normal vasculature (30), perhaps due to induction of endothelial expression by physiological rates of shear. In addition to its role as a vasodilator and platelet inhibitor, $\mathrm{PGI}_{2}$ appears to be important in protecting 
cardiomyocytes from oxidant stress (31). Likewise, COX-2 products appear to mediate the late phase of cardiac preconditioning (32). Indeed, prostanoids formed during inflammation are not exclusively proinflammatory (6). Both $\mathrm{PGE}_{2}$ and $\mathrm{PGI}_{2}$ downregulate the production of TNF- $\alpha$ and upregulate that of IL-10 through EP2 and EP4 and IP receptors, (15), consistent with speculation that the complex effects of inhibiting multiple prostanoids simultaneously somehow underlie the phenomenon of aspirin-induced asthma.

Clearly, these observations raise the possibility that compounds acting on specific receptors may offer advantages over upstream inhibitors. In contrast to deletion of COX-2 or, indeed, both COX isozymes, deletion of prostanoid receptors, with the exception of EP4, have not been associated with serious problems during development or in the perinatal period, and the mature animals appear normal under physiological conditions. A combination of pharmacological and genetic approaches may be needed to elucidate the different effects of receptor antagonism or of COX inhibition.

\section{Controversies regarding nuclear PG receptors}

There is now considerable interest in the putative nuclear actions of prostanoids (33). This interest stems from several reports proposing that prostanoids work as endogenous ligands for PPARs - 15 -deoxy- $\Delta 12,14$ $\mathrm{PGJ}_{2}$ (a dehydration metabolite of $\mathrm{PGD}_{2}$ ) as a ligand of PPAR $\gamma$, leukotriene $\mathrm{B} 4$ as a ligand for PPAR $\alpha$, and $\mathrm{PGI}_{2}$ analogs as ligands of PPAR $\delta$. However, the physiological role of prostanoids as PPAR activators has not been critically examined, partly because many COX inhibitors are themselves PPAR ligands and cannot be used to test the involvement of prostanoids in PPAR activation in vivo (34). The limitations to our current understanding are illustrated by a detailed consideration of $\mathrm{PGJ}_{2}$ and its derivatives, including 15-deoxy$\Delta 12,14-\mathrm{PGJ}_{2}$. These compounds were first identified in culture media containing $\mathrm{PGD}_{2}$, and later studies revealed that dehydration was carried out by slow catalysis of serum albumin in the culture medium.

It remains controversial whether $\mathrm{PGJ}_{2}$ is actually formed in vivo and, if so, to what extent. On the one hand, there is evidence for detection of it or its metabolites based on immunoassays in human urine and in body fluids, such as inflammatory exudates (6). However, these assays have not been validated by physicochemical methods. Recently, there has been a preliminary report of detecting the $\mathrm{PGJ}_{2}$ metabolite in human urine (35). However, little information on the assay was provided, and there remains a clear need to determine whether biologically active quantities of PGs of the J series are actually formed in vivo. It has been suggested that cyclopentenone PGs are so reactive that they might largely elude detection in body fluids such as plasma or urine. In any event, the quantities of exoge- nous $\mathrm{PGJ}_{2}$ ligands shown to activate PPARs are usually in the 5-10 micromolar range, whereas conventional PGs, acting via membrane receptors, are active in the nanomolar to femtomolar range, consistent with the amounts of these compounds actually formed in vivo. Thus, given that several polyunsaturated fatty acids can ligate PPARs at concentrations actually found in cells, we believe that the case for these dehydration products to function as physiological mediators remains unproven. Furthermore, these compounds are highly reactive electrophiles, due to the presence of an $\alpha, \beta$ unsaturated ketone, so they may crosslink to biomolecules, especially those with free SH residues. Calculations based on bound radioactivity suggest that more than $10^{5}$ proteins are modified, far exceeding the number of nuclear receptors (36). Thus, a broad range of activities - antiproliferative, antiviral, and anti-inflammatory effects, for example - might be anticipated when the synthetic compounds are applied in sufficient concentrations in vitro. Whether these activities occur in vivo remains a more controversial issue.

Less evidence supports a role for other eicosanoids as nuclear ligands. For example, $\mathrm{PGI}_{2}$ analogs can ligate PPAR $\delta$ in vitro, PGI synthase and PPAR $\delta$ colocalize in colonic tumors, and there is some evidence that ligation of PPAR $\delta$ by $\mathrm{PGI}_{2}$ is critical for implantation (37). However, while IP-deficient mice do not exhibit a reproductive defect (5), neither, reportedly, do mice lacking PPAR $\delta$ (38). Clarity may be afforded on this issue by comparing the phenotypes of mice lacking biosynthetic enzymes (e.g., PGI synthase) with those of mice carrying mutations in the relevant cell-surface receptors.

\section{Summary}

The availability of receptor knockouts and the use of expressed receptors to aid in the development of specific agonists and antagonists provide powerful tools for studying the biological importance of this group of bioactive lipids. This approach will be refined as tissuespecific inducible knockouts become available, and as mice carrying targeted mutations on a pure genetic background become more widely used. The combination of genetic approaches with the use of defined, specific pharmacological probes is likely to be extremely powerful.

Examples of polymorphic variation in some prostanoid receptors have already been reported (39), and the relevance of these observations to disease susceptibility and interindividual variations in drug response is likely to become increasingly clear. While the role of prostanoids and associated compounds as nuclear receptor ligands is a seductive possibility, conclusive evidence that such events actually occur at concentrations of the endogenous compounds attained in vivo remains to be established.

Note: Due to space constraints, a number of important references could not be included in this article. 


\section{Prostaglandins and their precursors} Garret A. FitzGerald, Series Editor

Interested readers can find a supplementary reading list at [www.jci.org/cgi/content/full/108/01/25/DC1].

1. Fitzpatrick, F.A., and Soberman, R. 2001. Regulated formation of eicosanoids. J. Clin. Invest. 107:1347-1351.

2. Narumiya, S., Sugimoto, Y., and Ushikubi, F. 1999. Prostanoid receptors: structures, properties, and functions. Physiol. Rev. 79:1193-1226.

3. Smith, E.M., Austin, S.C., Reilly, M.P., and FitzGerald, G.A. 2000. Internalization and sequestration of the human prostacyclin receptor. J. Biol. Chem. 275:32037-32045.

4. Kunapuli, P., Lawson, J.A., Rokach, J.A., Meinkoth, J.L., and FitzGerald, G.A. 1998. Prostaglandin $\mathrm{F}_{2 \alpha}\left(\mathrm{PGF}_{2 \alpha}\right)$ and the isoprostane, 8,12-iso-isoprostane $\mathrm{F}_{2 \alpha}$-III, induce cardiomyocyte hypertrophy: differential activation of downstream signaling pathways. J. Biol. Chem. 273:22442-22452.

5. Murata, T., et al. 1997. Altered pain perception and inflammatory response in mice lacking prostacyclin receptor. Nature. 388:678-682.

6. Gilroy, D.W., et al. 1999. Inducible cyclooxygenase may have anti-inflammatory properties. Nat. Med. 5:698-701.

7. Ushikubi, F., et al. 1998. Impaired febrile response in mice lacking the prostaglandin E receptor subtype EP3. Nature. 395:281-284.

8. Matsuoka, T., et al. 2000. Prostaglandin $\mathrm{D}_{2}$ as a mediator of allergic asthma. Science. 287:2013-2017.

9. Rocca, B., Spain, L., Pure, E., Patrono, C., and FitzGerald, G.A. 1999. Distinct and coordinated roles of prostaglandin $\mathrm{H}$-synthesis 1 and 2 in T-cell development. J. Clin. Invest. 103:1469-1477.

10. Hirai, H., et al. 2001. Prostaglandin D2 selectively induces chemotaxis in Thelper type 2 cells, eosinophils and basophils via seven-transmembrane receptor CRTH2. J. Exp. Med. 193:255-261.

11. Tilley, S.L., Coffman, T.M., and Koller, B.H. 2001. Mixed messages: modulation of inflammation and immune responses by prostaglandins and thromboxanes. J. Clin. Invest. 108:15-23.

12. Sakuma, Y., et al. 2000. Crucial involvement of the EP4 subtype of prostaglandin $\mathrm{E}$ receptor in osteoclast formation by proinflammatory cytokines in lipopolysaccharide. J. Bone Miner. Res. 15:218-227.

13. Miyaura, C., et al. 2000. Impaired bone resorption to prostaglandin E2 in prostaglandin E receptor EP4-knockout mice. J. Biol. Chem. 275:19819-19823.

14. Li, X., et al. 2000. Knockout of the murine prostaglandin EP2 receptor impairs osteoclastogenesis in vitro. Endocrinology. 141:2054-2061.

15. Shinomiya, S., et al. 2001. Regulation of TNF $\alpha$ and interleukin-10 production by prostaglandins $\mathrm{I}_{2}$ and $\mathrm{E}_{2}$ : studies with prostaglandin receptordeficient mice and prostaglandin E-receptor subtype-selective synthetic agonists. Biochem. Pharmacol. 61:1153-1160.

16. Smith, W.L., and Langenbach, R. 2001. Why there are two cyclooxygenase isozymes. J. Clin. Invest. 107:1491-1495.

17. Watanabe, K., et al 1999. Role of prostaglandin E receptor subtype EP1 in colon carcinogenesis. Cancer Res. 59:5093-5096.

18. Prescott, S.M. 2000. Is cyclooxygenase- 2 the alpha and the omega in cancer? J. Clin. Invest. 105:1511-1513.

19. Patrono, C., Patrignani, P., and García Rodríguez, L.A. 2001. Cyclooxygenase-selective inhibition of prostanoid formation: transducing biochemical selectivity into clinical read-outs. J. Clin. Invest. 108:7-13.

20. Thomas, D.W., et al. 1998. Coagulation defects and altered hemodynamic responses in mice lacking receptors for thromboxane A2. J. Clin. Invest. 102:1994-2001.

21. Habib, A., FitzGerald, G.A., and Maclouf, J. 1999. Phosphorylation of the thromboxane receptor $\alpha$, the predominant isoform expressed in human platelets. J. Biol. Chem. 274:2645-2651.

22. Miggin, S.M., and Kinsella, B.T. 1998. Expression and tissue distribution of the mRNAs encoding the human thromboxane $\mathrm{A}_{2}$ receptor (TP) isoforms. Biochim. Biophys. Acta. 1425:543-559.

23. Rocca, B., et al. 2000. Directed vascular expression of the thromboxane A2 receptor results in intrauterine growth retardation. Nat. Med. 6:219-221

24. Cayatte, A.J., et al. 2000. The thromboxane receptor antagonist S18886 but not aspirin inhibits atherogenesis in apo E-deficient mice: evidence that eicosanoids other than thromboxane contribute to atherosclerosis. Arterioscler. Thromb. Vasc. Biol. 20:1724-1728.

25. Kennedy, C.R., et al. 1999. Salt-sensitivity hypertension and reduced fertility in mice lacking the prostaglandin EP2 receptor. Nat. Med. 5:217-220.
26. Tilley, S.L., et al. 1999. Reproductive failure and reduced blood pressure in mice lacking the $\mathrm{E}$ prostaglandin $\mathrm{E} 2$ receptor. J. Clin. Invest. 103:1539-1545.

27. Kiriyama, M., Ushikubi, F., Kobayashi, T., and Narumiya, S. 1997. Ligand binding specificities of the eight types and subtypes of the mouse prostanoid receptors expressed in Chinese hamster ovary cells. Br. J. Pharmacol. 122:217-224.

28. Okada, Y., et al. 2000. Characterization of prostanoid receptors mediating contraction of the gastric fundus and ileum: studies using mice deficient in prostanoid receptors. Br. J. Pharmacol. 131:745-755.

29. McAdam, B.F., et al. 2000. Effect of regulated expression of human cyclooxygenase isoforms on eicosanoid and isoeicosanoid production in inflammation. J. Clin. Invest. 105:1473-1482.

30. McAdam, B.F., et al. 1999. Systemic biosynthesis of prostacyclin by cyclooxygenase: the human pharmacology of a selective inhibitor of COX2. Proc. Natl. Acad. Sci. USA. 96:272-277.

31. Adderly, S.R., and Fitzgerald, D.J. 1999. Oxidative damage of cardiomyocytes is limited by extracellular regulate kinases $1 / 2$-mediated induction of cyclooxygenase-2. J. Biol. Chem. 274:5038-5046.

32. Shimmura, K., et al. 2000. Cyclooxygenase- 2 mediates the cardioprotective effects of the late phase of ischemic preconditioning in conscious rabbits. Proc. Natl. Acad. Sci. USA. 97:10197-10202.

33. Forman, B.M., et al. 1995. 15-deoxy- $\Delta 12,14$-prostaglandin $\mathrm{J}_{2}$ is a ligand for the adipocyte determination factor PPAR $\gamma$. Cell. 83:803-812.

34. Lehman, J.M., Lenhard, J.M., Oliver, B.B., Ringold, G.M., and Kliewer, S.A. 1997. Peroxisome proliferator-activated receptors $\alpha$ and $\gamma$ are activated by indomethacin and other non-steroidal anti-inflammatory drugs. J. Biol. Chem. 272:3406-3410.

35. Thevenon, C., Guichardant, M., and Lagarde, M. 2001. Gas chromatographic-mass spectrometric measurement of 15 -deoxy-delta $(12,14)$ prostaglandin $\mathrm{J}_{2}$, the peroxisome proliferator-activated receptor gamma ligand, in urine. Clin. Chem. 47:768-770.

36. Narumiya, S., Ohno, K., Fukushima, M., and Fujiwara, M. 1987. Site and mechanism of growth inhibition by prostaglandins. III. Distribution and binding of prostaglandin $\mathrm{A}_{2}$ and $\Delta 12$-prostaglandin $\mathrm{J}_{2}$ in nuclei. J. Pharmacol. Exp. Ther. 242:306-311.

37. Lim, H., et al. 1999. Cyclo-oxygenase-2-derives prostacyclin mediates embryo implantation in the mouse via PPAR $\delta$. Genes Dev. 13:1561-1574.

38. Peters, J.M., et al. 2000. Growth, adipose, brain, and skin alterations resulting from targeted disruption of the mouse peroxisome proliferatoractivated receptor $\beta(\delta)$. Mol. Cell Biol. 20:5119-5128.

39. Unoki, M., et al. 2000. Association studies of 33 single nucleotide polymorphisms (SNPs) in 29 candidate genes for bronchial asthma: positive association a T924C polymorphism in the thromboxane $\mathrm{A}_{2}$ receptor gene. Hum. Genet. 106:440-446.

40. Hizaki, H., Segi, E., Sugimoto, Y., Hirose, M., et al. 1999. Abortive expansion of the cumulus and impaired fertility I mice lacking the prostaglandin E receptor subtype EP2. Proc. Natl. Acad. Sci. USA. 96:10501-10506

41. Audoly, L.P., et al. 1999. Identification of specific EP receptors responsible for the hemodynamic effects of PGE2. Am J. Physiol. 277:H924-H930.

42. Zhang, Y., et al. 2000. Characterization of murine vasopressor and vasodepressor prostaglandin E2 receptors. Hypertension. 35:1129-1134.

43. Sheller, J.R., Mitchell, D., Meyrick, B., Oates, J., and Breyer, R. 2000. EP2 receptor mediates bronchodilation by $\mathrm{PGE}_{2}$ in mice. J. Appl. Physiol. 88:2214-2218.

44. Takeuchi, K., et al. 1999. Impaired duodenal bicarbonate secretion and mucosal integrity in mice lacking prostaglandin E-receptor subtype EP3. Gastroenterol. 117:1128-1135.

45. Fleming, E.F., et al. 1998. Urinary concentrating function in mice lacking EP3 receptors for prostaglandin E2. Am. J. Physiol. 275:F955-F961.

46. Nguyen, M.T., et al. 1997. The prostaglandin receptor EP4 triggers remodeling of the cardiovascular system at birth. Nature. 390:78-81.

47. Segi, E., et al. 1998. Patent ductus arteriosus and neonatal death in prostaglandin receptor deficient mice. Biochem. Biophys. Res. Commun. 246:7-12.

48. Sugimoto, Y., et al. 1997. Failure of parturition in mice lacking the prostaglandin F receptor. Science. 277:681-684.

49. Ueno, A., et al. 2000. Intrinsic prostacyclin contributes to exudation induced by bradykinin or carrageenin: a study on the paw edema induced in IP-receptor-deficient mice. Life Sci. 66:155-160. 